TITLE:

\title{
Structural and mutational analysis of amino acid residues involved in ATP specificity of Escherichia coli acetate kinase.
}

\author{
$\operatorname{AUTHOR}(\mathrm{S})$ :
}

Yoshioka, Aya; Murata, Kousaku; Kawai, Shigeyuki

\section{CITATION:}

Yoshioka, Aya ... [et al]. Structural and mutational analysis of amino acid residues involved in ATP specificity of Escherichia coli acetate kinase.. Journal of bioscience and bioengineering 2014, 118(5): 502-507

\section{ISSUE DATE:}

2014-05-23

URL:

http://hdl.handle.net/2433/198558

\section{RIGHT:}

(c) 2014 The Society for Biotechnology, Japan. Published by Elsevier B.V. Licensed under the Creative Commons Attribution-NonCommercial-NoDerivatives 4.0 International http://creativecommons.org/licenses/by-nc-nd/4.0/. NOTICE: this is the author's version of a work that was accepted for publication in Journal of Bioscience and Bioengineering. Changes resulting from the publishing process, such as peer review, editing, corrections, structural formatting, and other quality control mechanisms may not be reflected in this document. Changes may have been made to this work since it was submitted for publication. A definitive version was subsequently published in Journal of Bioscience and Bioengineering, Volume 118, Issue 5, Pages 502-507. doi:10.1016/j.jbiosc.2014.04.014.; This is not the published version. Please cite only the published version.; この論文は出版社版でありません。引用の際には出版社版

をご確認ご利用ください。 
1 Title: Structural and mutational analysis of amino acid residues involved in ATP

2 specificity of $E$. coli acetate kinase

3

4 Short title: Specificity to ATP of acetate kinase

5

6 Author names: Aya Yoshioka, Kousaku Murata, Shigeyuki Kawai *

7

8 Affiliations: Laboratory of Basic and Applied Molecular Biotechnology, Division

9 of Food Science and Biotechnology, Graduate School of Agriculture, Kyoto

10 University, Uji, Kyoto 611-0011, Japan

11

12 *To whom correspondence should be addressed.

13 Tel: +81 77438 3768; Fax: +81 77438 3767; E-mail: kawais@kais.kyoto-u.ac.jp. 


\section{Abstract}

Acetate kinase (AK) generally utilizes ATP as a phosphoryl donor, but AK

20 from Entamoeba histolytica (PPi-ehiAK) uses PPi, not ATP, and is PPi-specific.

21 The determinants of the phosphoryl donor specificity are unknown. Here, we

22 inferred 5 candidate amino acid residues associated with this specificity, based on

23 structural information. Each candidate residue in Escherichia coli ATP-specific

24 AK (ATP-ecoAK), which is unable to use PPi, was substituted with the respective

25 PPi-ehiAK amino acid residue. Each variant ATP-ecoAK had an increased $K_{\mathrm{m}}$ for

26 ATP, indicating that the 5 residues are the determinants for the specificity to ATP

27 in ATP-ecoAK. Moreover, Asn-337 of ATP-ecoAK was shown to be particularly

28 significant for the specificity to ATP. The 5 residues are highly conserved in 2,625

29 PPi-ehiAK homologs, implying that almost all organisms have ATP-dependent,

30 rather than $\mathrm{PPi}$-dependent, $\mathrm{AK}$.

$32 \quad$ Key words

33 PPi; ATP; acetate kinase; Entamoeba histolytica; Methanosarcina thermophila 


\section{Introduction}

Acetate kinase (AK) is a critical enzyme for central carbon metabolism in bacteria and archea (1). ATP-dependent AK catalyzes the phosphorylation of acetate to produce acetyl phosphate by utilizing ATP as a phosphoryl donor and also catalyzes the reverse reaction (2). This enzyme has been purified and characterized from various archaea and bacteria, including Methanosarcina thermophila, Escherichia coli, Thermotoga maritima, and Lactobacillus sanfranciscensis (3-7). The three-dimensional structure of AK of M. thermophila (mthAK) has been solved (8) and several key catalytic residues have been identified based on the tertiary structural information and site-directed mutagenesis (8-15).

Flower et al. have recently shown that the AK of the amitochondriate protist Entamoeba histolytica (PPi-ehiAK) is a novel PPi-dependent AK that phosphorylates acetate using PPi, not ATP, as a phosphoryl donor (16). The three-dimensional structure of PPi-ehiAK has been solved and the substrate-binding site of PPi-ehiAK has been compared with that of mthAK, although without a biochemical verification (17). Despite the differences in phosphoryl donor specificity, the primary sequences of AK enzymes are highly conserved $(8,17)$.

The significance of AK in central carbon and energy metabolism indicates the importance of understanding the determinants of the specificity of AK for phosphoryl donors (ATP and PPi). Such understanding is also important in a structural biology context, given the structural overlap between ATP and PPi (Fig. 1). In this study, we verified that PPi-ehiAK is specific for PPi, and does not 
60 accept ATP; and that E. coli AK (ATP-ecoAK) is specific for ATP, and not for PPi.

61 We then identified the amino acid determinants of the specificity to ATP of ATP-ecoAK based on a comparison of the tertiary structures of mthAK and PPi-ehiAK and the primary structures of mthAK, PPi-ehiAK, and ATP-ecoAK,

64 combined with site-directed mutagenesis of ATP-ecoAK.

MATERIALS AND METHODS

Expression of PPi-ehiAK PPi-ehiAK (KEGG ID, ehi, EHI_170010) with

optimized codon usage for E. coli was synthesized at Operon (Tokyo, Japan)

70 (Supplementary Fig. S1). PPi-ehiAK was amplified by PCR using the synthesized gene as a template with primers AK_pQE_B_F (5'-TCA CCA TCA CGG ATC CAT GTC TAA TGT GCT GAT TTT C-3', with the BamHI site underlined) and AK_pQE_S_R (5’-GCT GCA GGT CGA CCC TTA AAA CTG GAA TAA TTC TTT C-3'). The PCR product was inserted into BamHI/SmaI sites in pQE-80L with In-Fusion (Clontech, Otsu, Japan), yielding pMK3549. E. coli MK3648 strain was obtained by transforming E. coli NovaBlue (Novagen) with pMK3549 and plasmid pLysSRARE (Novagen). PPi-ehiAK is expressed as a N-terminally His-tagged protein in E. coli MK3648.

For expression of PPi-ehiAK, a fresh single colony of MK3648 strain that had 80 just been transformed was inoculated into $10 \mathrm{~mL}$ LB medium supplemented with $81100 \mu \mathrm{g} / \mathrm{mL}$ ampicillin, $34 \mu \mathrm{g} / \mathrm{mL}$ chloramphenicol, and $12.5 \mu \mathrm{g} / \mathrm{mL}$ tetracycline.

82 After culturing at $37^{\circ} \mathrm{C}$ aerobically overnight, the culture was transferred to the 83 same medium $(250 \mathrm{~mL})$ and cultured at $37^{\circ} \mathrm{C}$ aerobically overnight. This culture 
84 was again transferred to the same medium $(4.5 \mathrm{~L}, 500 \mathrm{~mL}$ in a $2.0 \mathrm{~L}$ Sakaguchi

85 flask) and cultivation was continued at $37^{\circ} \mathrm{C}$ aerobically until $A_{600}$ reached 0.4.

Then, isopropyl-1- $\beta$-D-thiogalactopyranoside was added to $1 \mathrm{mM}$ and cultivation was continued further at $37^{\circ} \mathrm{C}$ aerobically for $1 \mathrm{~h}$.

Purification of PPi-ehiAK MK3648 cells overexpressing PPi-ehiAK were collected by centrifugation at $8,500 \mathrm{~g}$ for $10 \mathrm{~min}$, suspended in $50 \mathrm{~mL}$ of $20 \mathrm{mM}$ HEPES-NaOH (pH 7.5), and disrupted by sonication at $4^{\circ} \mathrm{C}$ for 15 min with an Insonator 201M (Kubota, Tokyo, Japan). After centrifugation at 20,000 $g$ for 10

93 min, the clear supernatant was used as the cell extract containing PPi-ehiAK. This 94 cell extract was applied to a Talon Metal Affinity Resin column $(2.5 \times 4.5 \mathrm{~cm})$ 95 (Clontech) equilibrated with 20 mM HEPES-NaOH (pH 7.5). After washing with $150 \mathrm{~mL}$ of $20 \mathrm{mM}$ HEPES-NaOH (pH 7.5) containing $30 \mathrm{mM}$ imidazole and 300 mM NaCl, PPi-ehiAK was eluted with 150 mL of 20 mM HEPES-NaOH (pH 7.5) containing $150 \mathrm{mM}$ imidazole and $300 \mathrm{mM} \mathrm{NaCl}$. The fractions containing PPi-ehiAK were combined, dialyzed against $20 \mathrm{mM}$ HEPES-NaOH $(\mathrm{pH} 7.5)$ at 100 $4^{\circ} \mathrm{C}$ overnight, and used as the purified PPi-ehiAK. If necessary, the purified enzyme was concentrated by 3-fold with a Centriprep 10K instrument (Millipore).

Expression of ATP-ecoA For expression of ATP-ecoAK (KEGG ID, ecj, 104 Y75_p2262), E. coli K12 AG1 ASKA GFP-free strain (MK3814 strain), which 105 contained plasmid pMK3814 (pCA24N carrying the ATP-ecoAK gene without GFP) (18) and was stored at $-80^{\circ} \mathrm{C}$ in the presence of $17 \% \mathrm{v} / \mathrm{v}$ glycerol, was inoculated into $20 \mathrm{~mL}$ LB medium supplemented with $34 \mu \mathrm{g} / \mathrm{mL}$ chloramphenicol 
and cultured at $37^{\circ} \mathrm{C}$ aerobically overnight. This culture was transferred to the same medium (700 mL, $350 \mathrm{~mL}$ in a $500 \mathrm{~mL}$ Sakaguchi flask) and cultivation was continued at $37^{\circ} \mathrm{C}$ aerobically until $A_{600}$ reached 0.6. Then, isopropyl-1- $\beta$-D-thiogalactopyranoside was added to $0.1 \mathrm{mM}$ and cultivation was continued at $37^{\circ} \mathrm{C}$ aerobically for $3 \mathrm{~h}$. Purification and dialysis were conducted as described for PPi-ehiAK above, but with use of $10 \mathrm{mM}$ Tris-HCl (pH 8.0) instead of 20 mM HEPES-NaOH (pH 7.5).

\section{Expression of variant ATP-ecoAK Site-directed mutagenesis was performed} using inverse PCR followed by DpnI treatment using pMK3814 as a template. The variant ATP-ecoAK plasmids were confirmed by DNA sequencing. These plasmids were introduced into the $E$. coli DH5a strain and each variant ATP-ecoAK was expressed and purified as described below for ATP-ecoAK.

Assay of AK activity The AK activity in the acetyl phosphate-forming direction was assayed at $30^{\circ} \mathrm{C}$ using a hydroxamate assay that detects formation of acetyl phosphate $(2,19)$. The assay utilizes the reaction of acetyl phosphate with hydroxylamine to form acetyl hydroxamate, which forms a colored complex with trivalent iron. The reaction mixture (333 $\mu \mathrm{L})$ comprised $10 \mathrm{mM}$ phosphoryl donor, 150 mM HEPES-NaOH (pH 6.5), 200 mM potassium acetate, $10 \mathrm{mM} \mathrm{MgCl}$, 700 mM hydroxylamine hydrochloride, with the $\mathrm{pH}$ adjusted to $\mathrm{pH} 6.5$ with $\mathrm{KOH}$ just before use, and an appropriate amount of AK. The reaction was initiated by addition of $\mathrm{AK}$ and terminated by addition of $333 \mu \mathrm{L}$ of $10 \%$ trichloroacetic acid, followed by addition of $333 \mu \mathrm{L}$ of $2.5 \% \mathrm{FeCl}_{3}$ in $2.0 \mathrm{~N} \mathrm{HCl}$. After incubation for 
$1325 \mathrm{~min}$, the absorbance at $540 \mathrm{~nm}\left(A_{540}\right)$ was measured and defined as ${ }^{1} A_{540}$. PPi 133 (Nakalai Tesque, Kyoto, Japan) and ATP (Wako Pure Chemical) were used as 134 phosphoryl donors. AK was diluted with 20 mM HEPES-NaOH (pH 7.5) or 10 $135 \mathrm{mM}$ Tris-HCl $(\mathrm{pH}$ 8.0) if required. Control reactions were conducted without a 136 phosphoryl donor and the resultant $A_{540}$ was defined as ${ }^{2} A_{540}$. AK activity was 137 calculated as $\Delta A_{540}\left({ }^{1} A_{540}-{ }^{2} A_{540}\right)$.

138 The AK activity in the PPi-forming direction was assayed at $30^{\circ} \mathrm{C}$ by detecting 139 formation of PPi using a PPiLight ${ }^{\mathrm{TM}}$ inorganic pyrophosphate assay (Lonza, Basel, 140 Switzerland) (20, 21). This assay utilizes the reactions of PPi with AMP to form 141 ATP, and of luciferase producing light from the newly formed ATP. The reaction mixture $(50 \mu \mathrm{L})$ comprised $100 \mathrm{mM}$ HEPES-NaOH (pH 6.5), $20 \mathrm{mM}$ potassium 143 phosphate (Pi) (pH 7.0), $20 \mathrm{mM} \mathrm{MgCl}_{2}, 1.5 \mathrm{mM}$ acetyl phosphate, and an 144 appropriate amount of AK. The reaction was initiated by addition of AK and 145 terminated by boiling for $5 \mathrm{~min}$. After the reaction, $20 \mu \mathrm{L}$ of PPiLight ${ }^{\mathrm{TM}}$ 146 Converting Reagent was added to the reaction mixture ( $40 \mu \mathrm{L})$ with appropriate 147 dilution with distilled water (e.g., 50-fold dilution for the PPi-ehiAK reaction). 148 After incubation at room temperature for $30 \mathrm{~min}, 20 \mu \mathrm{L}$ of PPiLight ${ }^{\mathrm{TM}}$ Detection 149 Reagent was added and the mixture $(60 \mu \mathrm{L})$ was further incubated at room 150 temperature for 30 min. Luminescence of the mixture was then read with a $0.1 \mathrm{~s}$ 151 integration time and the relative luminescent unit (RLU) value was measured as $152{ }^{1}$ RLU. Control reactions were conducted without AK and the resultant RLU was 153 defined as ${ }^{2}$ RLU. AK activity in the PPi-forming direction was calculated as $154 \Delta \mathrm{RLU}\left({ }^{1} \mathrm{RLU}-{ }^{2} \mathrm{RLU}\right)$ using an authentic PPi as a standard.

155 One unit (U) of enzyme activity was defined as $1 \mu \mathrm{mol}$ acetyl phosphate 
156 produced in $1 \mathrm{~min}$ at $30^{\circ} \mathrm{C}$ and specific activity was expressed in $\mathrm{U} / \mathrm{mg}$ protein.

157 Protein concentrations were determined by the method of Bradford (22) with

158 bovine serum albumin as a standard.

Differential scanning fluorimetry (DSF) Interaction between AK and substrate (ATP or PPi) was assessed by DSF (23) using a MyiQ2 real-time PCR instrument (Bio-Rad). The fluorescence of SYPRO Orange (Invitrogen) was monitored using filters provided with the PCR instrument (excitation at $492 \mathrm{~nm}$ and emission at $610 \mathrm{~nm})$. The reaction mixture (20 $\mu \mathrm{l})$ comprised $5 \mu \mathrm{g} \mathrm{AK,} 20$ mM Tris-HCl (pH 7.5), 1000-fold diluted SYPRO Orange, and each substrate (1.0 $\mathrm{mM}$ ATP, $5.0 \mathrm{mM} \mathrm{PPi}$ ). This mixture was subjected to a temperature increase from 20 to $95^{\circ} \mathrm{C}$ by $0.5^{\circ} \mathrm{C} / \mathrm{cycle}$ for a total of 141 cycles. The fluorescence profile was obtained by plotting the relative fluorescence unit (RFU) value at each temperature. The profile was analyzed using iQ5 (Bio-Rad) and the midpoint of

170 the increase in the profile was defined as the melting temperature $\left(T_{\mathrm{m}}\right)$.

Elution of AKs from an ATP-agarose column Purified ATP-ecoAK in 10 $\mathrm{mM}$ Tris-HCl (pH 8.0) containing $10 \mathrm{mM} \mathrm{MgCl}_{2}(500 \mu \mathrm{L}, 1.0 \mathrm{mg} / \mathrm{mL})$ was applied to an ATP-agarose column (100 $\mu \mathrm{L})$ (Sigma-Aldrich) equilibrated with 10

$175 \mathrm{mM}$ Tris-HCl (pH 8.0) containing $10 \mathrm{mM} \mathrm{MgCl}_{2}$. The column was allowed to 176 stand for $1 \mathrm{~h}$ at $4^{\circ} \mathrm{C}$ for binding to take place. After incubation, the column was washed with $2 \mathrm{~mL}$ of $10 \mathrm{mM}$ Tris- $\mathrm{HCl}(\mathrm{pH}$ 8.0) containing $10 \mathrm{mM} \mathrm{MgCl}$. Next, $178500 \mu \mathrm{L}$ of $50 \mathrm{mM}$ ATP (pH 7.0) containing $10 \mathrm{mM} \mathrm{MgCl}_{2}$ was added and the 179 column was incubated for $30 \mathrm{~min}$ at $4^{\circ} \mathrm{C}$, followed by elution of ATP-ecoAK. In 
180 the case of purified PPi-ehiAK in $20 \mathrm{mM}$ HEPES-NaOH (pH 7.5) containing 10 $\mathrm{mM} \mathrm{MgCl}_{2}(500 \mu \mathrm{L}, 1.0 \mathrm{mg} / \mathrm{mL})$, the application, washing, and elution procedures were the same as those for ATP-ecoAK, but with use of $20 \mathrm{mM}$ HEPES-NaOH (pH 7.5) instead of 10 mM Tris-HCl (pH 8.0).

SDS-PAGE SDS-PAGE was performed on 12.5\% acrylamide gels.(24)

Proteins in the gel were visualized with Coomassie brilliant blue R-250.

\section{RESULTS}

Purification and phosphoryl donor specificity of PPi-ehiAK and ATP-ecoAK An PPi-ehiAK gene with optimized codon usage for E. coli was synthesized (Fig. S1), cloned in pQE-80L, and expressed as a N-terminally

193 His-tagged protein in E. coli. The purified PPi-ehiAK protein was $44 \mathrm{kDa}$ on 194 SDS-PAGE, in agreement with the calculated molecular mass (44 kDa; His-tag, 1 $195 \mathrm{kDa}+$ PPi-ehiAK, $43 \mathrm{kDa}$ ) (Fig. S2). Kinetic constants showed that the purified 196 PPi-ehiAK utilized PPi, but not ATP, as a phosphoryl donor (Table 1). Native 197 ATP-ecoAK purified from E. coli utilizes ATP (7), but the strict phosphoryl donor 198 specificity of ATP-ecoAK is unknown. To examine this issue, a N-terminally 199 His-tagged ATP-ecoAK was also expressed in E. coli. The purified ATP-ecoAK 200 was shown to be specific for ATP, and not to use PPi as a phosphoryl donor (Table 201 1). 
204 To probe the structural determinants of the phosphoryl donor specificity of AK, 205 we inspected the substrate-binding site of the tertiary structure of mthAK 206 complexed with acetate and $\mathrm{ADP}_{-} \mathrm{AlF}_{3}$ (9). Noted that although mthAK utilizes 207 ATP for the phosphorylation of acetate (5), if mthAK uses PPi or not has not been 208 experimentally confirmed. We found 12 residues located within $4.0 \AA$ of ADP: 209 Leu-209, Gly-210, Asn-211, Asp-283, Phe-284, Arg-285, Ala-330, Gly-331, 210 Ile-332, Asn-335, Ser-336, and Arg-362. Among these residues, 5 (Asn-211, 211 Ala-330, Gly-331, Ile-332, and Asn-335 of mthAK) are conserved in ATP-ecoAK, 212 but not in PPi-ehiAK, based on a multiple sequence alignment (Fig. 2). This 213 suggests that these residues are candidates for determinants of the phosphoryl 214 donor specificity. Four of these 5 residues (Asn-211, Gly-331, Ile-332, and 215 Asn-335) form hydrogen bonds and van der Waals contacts to ADP (Table S1). Of 216 the other 7 residues among the original 12 in mthAK, two (Ser-336 and Arg-362) 217 are not conserved and 5 (Leu-209, Gly-210, Asp-283, Phe-284, and Arg-285) have 218 defined roles $(8,12)$ and are conserved in both ATP-ecoAK and PPi-ehiAK (Fig. $2192)$

220 Among the 5 candidate residues, Asn-211, Gly-331, and Ile-332 of mthAK 221 have been proposed to bind to the $\beta$-phosphate, $\alpha$-phosphate, and adenine base of 222 ADP, respectively, although this has not been confirmed experimentally (Fig. 3) 223 (8). Ala-330 is located close to the $\beta$-phosphate and Asn-335 interacts with the 224 adenine base of ADP (Table S1), suggesting that Ala-330 and Asn-335 are key 225 residues in recognition of ATP in mthAK (Fig. 3). Superposition of the 226 substrate-binding site of the tertiary structure of mthAK complexed with acetate

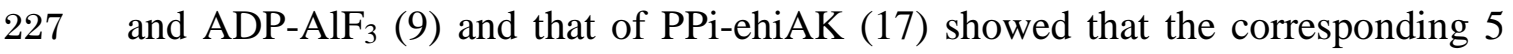


228 residues in PPi-ehiAK would occlude the ATP-binding cleft of mthAK and thus

229 could reject binding of ATP to PPi-ehiAK (Fig. 3). Consistent with this

230 observation, Gln-323 and Met-324 of PPi-ehiAK have previously been proposed

231 to occlude the ATP-binding cleft (17).

232

233 Kinetic analyses of variant ATP-ecoAKs The candidate residues in Ala-330/Gly-332/Asp-322, Gly-331/Gly-333/Gln-323, Ile-332/Ile-334/Met-324, and Asn-335/Asn-337/Glu-327 (Figs. 2 and 3). To examine the roles of these residues in phosphoryl donor specificity, each candidate residue in ATP-ecoAK, which is specific for ATP (Table 1), was substituted with the respective PPi-ehiAK residue and the variant ATP-ecoAKs were purified (Fig. S2). All 5 variant ATP-ecoAKs exhibited increased $K_{\mathrm{m}}$ and decreased $V_{\max }$ for ATP (Table 1), indicating that these residues are the determinants for the specificity to ATP in

242 ATP-ecoAK. Notably, $K_{\mathrm{m}}$ for ATP of ATP-ecoAK N337E increased 46-fold 243 relative to ATP-ecoAK, showing a critical role of ATP-ecoAK Asn-337 in ATP 244 binding. Thus, Glu-327 of PPi-ehiAK may be a particularly potent determinant of 245 rejection of ATP and promotion of PPi specificity in PPi-ehiAK (Fig. 3, Table 1). 246 Each variant ATP-ecoAK showed no PPi-dependent AK activity for both acetyl 247 phosphate and PPi formation (Tables 1 and S2).

249 Analyses of substrate binding to AKs Affinities of PPi-ehiAK, ATP-ecoAK, 250 and variant ATP-ecoAKs for each ATP and PPi were analyzed using DSF based on 251 changes in protein stability upon ligand binding (23). The fluorescence of SYPRO 
252 Orange bound to denatured proteins was measured during heat treatment from 25 to $95^{\circ} \mathrm{C} . T_{\mathrm{m}}$ values of AKs in the absence and presence of substrates were

254 determined as the midpoint of the increase in the fluorescence profile (Table 2).

255 The difference in $T_{\mathrm{m}}$ of native ATP-ecoAK in the presence and absence of ATP 256 ( $\Delta T_{\mathrm{m}}$ АTP) was $13^{\circ} \mathrm{C}$, suggesting that the thermal stability of ATP-ecoAK was increased by the binding of ATP. In contrast, $\Delta T_{\mathrm{m}}$ ATP of PPi-ehiAK could not be measured, concordant with the absence of ATP-dependent activity of PPi-ehiAK (Table 1). $\Delta T_{\mathrm{m} \text { ATP }}$ of all variant ATP-ecoAKs was decreased significantly relative to that of native ATP-ecoAK, in agreement with the decreased activity of variant 261 ATP-ecoAK and supporting the roles of the 5 residues as determinants of 262 specificity for ATP. The reason for the greatly decreased $\Delta T_{\mathrm{m}}$ ATP for ATP-ecoAK G332D remains to be clarified.

To confirm the ATP-binding data, ATP-ecoAK, ATP-ecoAK N337E, and PPi-ehiAK were added to an ATP-agarose column, washed, and eluted with 50 mM ATP containing $10 \mathrm{mM} \mathrm{MgCl}_{2}$. As expected, ATP-ecoAK was eluted by ATP 267 after complete washing, while PPi-ehiAK was not eluted (Fig. S3), in agreement with the absence of ATP-dependent activity of PPi-ehiAK and the DSF results

269 (Tables 1 and 2). Slight elution occurred for ATP-ecoAK N337E (Fig. S3), which 270 had a significantly increased $K_{\mathrm{m}}$ for ATP (Table 1 ). This confirms that the affinity 271 of ATP-ecoAK N337E for ATP was decreased by substitution of Asn-337 to Glu. $272 \Delta T_{\mathrm{m}}$ PPi of PPi-ehiAK was 1.7 , indicating the expected binding of PPi to 273 PPi-ehiAK (Table 2). Unexpectedly, $\Delta T_{\mathrm{m} \text { PPi }}$ of ATP-ecoAK was 4.4, suggesting 274 that PPi can bind to ATP-ecoAK, although ATP-ecoAK cannot utilize PPi as a 275 phosphoryl donor for catalysis (Table 1). $\Delta T_{\mathrm{m} \text { PPi }}$ of ATP-ecoAK was not markedly 
276 reduced upon substitution of each candidate residue, especially Gly-333, Ile-334, 277 and Asn-337, compared to the effects on $\Delta T_{\mathrm{m} \text { ATP }}$ (Table 2). This may reflect the 278 role of these residues in binding to adenosine of ADP, and thus not to PPi (Fig. 3). 279 Substitution of Asn-213 or Gly-332 of ATP-ecoAK reduced $\Delta T_{\mathrm{m}}$ PPi of 280 ATP-ecoAK. This is in agreement with the putative binding of Asn-213 of 281 ATP-ecoAK to the ß-phosphate of ADP and putative equivalent binding of 282 Asn-211 of mthAK to the PPi moiety. Gly-322 of ATP-ecoAK is located close to 283 the ß-phosphate of ADP, and Ala-330 of mthAK may similarly be located close to 284 PPi (Fig. 3).

285 Asn-213 and Gly-332 of ATP-ecoAK correspond to Thr-201 and Asp-322 of 286 PPi-ehiAK respectively (Figs. 2 and 3). To examine the involvement of Thr-201 287 and Asp-322 of PPi-ehiAK in utilization of PPi, we focused on the residues 288 (Leu-326 and Asn-376) of PPi-ehiAK that interact with Thr-201 and Asp-322 289 (Table S3, Fig. S4). Leu-326 and Asn-376 of PPi-ehiAK correspond to Glu-336 290 and Thr-385 of ATP-ecoAK. Glu-336, Thr-385, Asn-213 and Gly-332 of 291 ATP-ecoAK were substituted with the respective PPi-ehiAK residues (Leu-326, 292 Asn-376, Thr-201, and Asp-322) to give a quadruple ATP-ecoAK variant 293 (ATP-ecoAK N213T G332D E336L T385N) that had the possibility of utilizing 294 PPi (Fig. S2). However, this variant could not utilize PPi and $\Delta T_{\mathrm{m}}$ PPi was not 295 increased by these substitutions. $V_{\max }$ and $k_{\text {cat }}$ for ATP were significantly decreased 296 in the quadruple variant (Table 1) and $\Delta T_{\mathrm{m} \text { ATP }}$ was markedly decreased (Table 2). 297 
The results of the study show that PPi-ehiAK is specific for PPi and does not utilize ATP (16), while ATP-ecoAK is specific for ATP and does not use PPi. Based on the tertiary and primary structures and catalytic and binding activities of variant enzymes, we identified 5 residues (Asn-213, Gly-332, Gly-333, Ile-334, and Asn-337 in ATP-ecoAK) as determinants of specificity to ATP in ATP-ecoAK.

ATP-ecoAK N337E exhibited a 46-fold increase in $K_{\mathrm{m}}$ for ATP relative to ATP-ecoAK, indicating that Asn-337 of ATP-ecoAK is the most crucial for the specificity to ATP and suggesting that the corresponding Asn-335 in mthAK is also crucial for binding to ATP and that the corresponding Glu-327 in PPi-ehiAK 309 is possibly important for rejection of ATP in PPi-ehiAK. The basis of the role of 310 Asn-337 of ATP-ecoAK in accepting ATP was examined using molecular and electrostatic surfaces of the ADP-binding sites of mthAK N335E and mthAK, for which a tertiary structure has been solved (9). The ADP-binding cleft is negatively charged in mthAK N335E, but positively charged in mthAK (Fig. S5). There was no clear structural change in the molecular surface of mthAK N335E that could have resulted in rejection of ATP. Thus, the negatively charged ATP-binding site in ATP-ecoAK N337E may explain the weaker affinity for ATP.

To get further insight into the substrate specificity of AKs, we examined how

318 the 5 key residues are distributed in the primary structures of AKs. A total of 319 2,625 proteins homologous to PPi-ehiAK from all species with sequenced 320 genomes were found using BLASTP (25). Among the homologs, the 5 key 321 residues of mthAK/ATP-ecoAK/PPi-ehiAK (Asn-211/Asn-213/Thr-201, 322 Ala-330/Gly-332/Asp-322, Gly-331/Gly-333/Gln-323， Ile-332/Ile-334/Met-324, 323 and Asn-335/Asn-337/Glu-327) are highly conserved as Asn, Ala or Gly, Gly, Ile, 
324 and Asn, respectively (Table 3). These are mthAK- and ATP-ecoAK-specific 325 residues, which suggests that almost all organisms have ATP-dependent AKs. In 326 contrast, 10 PPi-ehiAK homologs, including PPi-ehiAK, possess the 327 PPi-ehiAK-specific residue (Glu) in the most crucial position determining the 328 phosphoryl donor specificity (corresponding to Asn-335/Asn-337/Glu-327 in 329 mthAK/ATP-ecoAK/PPi-ehiAK) (Table 3). However, except for PPi-ehiAK and 330 the PPi-ehiAK homolog from Entamoeba dispar, which is closely related to $E$. 331 histolytica, 8 of these homologs have no PPi-ehiAK-specific residues in the other 3324 positions (corresponding to Thr-201, Asp-322, Gln-323, and Met-324 in 333 PPi-ehiAK) (Table S4). This suggests that only the PPi-ehiAK homolog from E. 334 dispar is PPi-dependent. The phosphoryl donor specificities of the other 8 335 PPi-ehiAK homologs remain to be clarified.

\section{ACKNOWLEDGEMENTS}

338 We thank the National Bio Resource Project (NIG, Japan) for providing the $E$. 339 coli K12 AG1 ASKA GFP-free strain. This work was partially supported by the 340 Funding Program for Next Generation World-Leading Researchers (to S. K.). 


\section{REFERENCES}

344 1. Thauer, R. K., Moller-Zinkhan, D., and Spormann, A. M.:

345 Biochemistry of acetate catabolism in anaerobic chemotrophic bacteria,

346 Annu. Rev. Microbiol., 43, 43-67 (1989).

347 2. Rose, I. A.: Acetate kinase of bacteria (acetokinase), Methods. Enzymol., 1, 591-595 (1955).

349 3. Matsuyama, A., Yamamoto, H., and Nakano, E.: Cloning, expression, and nucleotide sequence of the Escherichia coli K-12 ackA gene, J. Bacteriol., 171, 577-580 (1989). characterization of acetate kinase from Lactobacillus sanfranciscensis, Microbiol. Res., 156, 267-277 (2001).

5. Aceti, D. J., and Ferry, J. G.: Purification and characterization of acetate kinase from acetate-grown Methanosarcina thermophila. Evidence for regulation of synthesis, J. Biol. Chem., 263, 15444-15448 (1988).

358 6. Bock, A. K., Glasemacher, J., Schmidt, R., and Schonheit, P.: Purification and characterization of two extremely thermostable enzymes, 1861-1867 (1999)

363 7. Fox, D. K., and Roseman, S.: Isolation and characterization of homogeneous acetate kinase from Salmonella typhimurium and Escherichia coli, J. Biol. Chem., 261, 13487-13497 (1986). 
8. Buss, K. A., Cooper, D. R., Ingram-Smith, C., Ferry, J. G., Sanders, D. A., and Hasson, M. S.: Urkinase: Structure of acetate kinase, a member of the ASKHA superfamily of phosphotransferases, J. Bacteriol., 183, 680-686 (2001).

9. Gorrell, A., Lawrence, S. H., and Ferry, J. G.: Structural and kinetic analyses of arginine residues in the active site of the acetate kinase from Methanosarcina thermophila, J. Biol. Chem., 280, 10731-10742 (2005).

10. Ingram-Smith, C., Barber, R. D., and Ferry, J. G.: The role of histidines in the acetate kinase from Methanosarcina thermophila, J. Biol. Chem., 275, 33765-33770 (2000).

11. Ingram-Smith, C., Gorrell, A., Lawrence, S. H., Iyer, P., Smith, K., and Ferry, J. G.: Characterization of the acetate binding pocket in the Methanosarcina thermophila acetate kinase, J. Bacteriol., 187, 2386-2394 (2005).

12. Miles, R. D., Iyer, P. P., and Ferry, J. G.: Site-directed mutational analysis of active site residues in the acetate kinase from Methanosarcina thermophila, J. Biol. Chem., 276, 45059-45064 (2001).

13. Miles, R. D., Gorrell, A., and Ferry, J. G.: Evidence for a transition state analog, MgADP-aluminum fluoride-acetate, in acetate kinase from Methanosarcina thermophila, J. Biol. Chem., 277, 22547-22552 (2002).

14. Singh-Wissmann, K., Ingram-Smith, C., Miles, R. D., and Ferry, J. G.: Identification of essential glutamates in the acetate kinase from Methanosarcina thermophila, J. Bacteriol., 180, 1129-1134 (1998).

15. Singh-Wissmann, K., Miles, R. D., Ingram-Smith, C., and Ferry, J. G.: 
Identification of essential arginines in the acetate kinase from Methanosarcina thermophila, Biochemistry (Mosc). 39, 3671-3677 (2000).

16. Fowler, M. L., Ingram-Smith, C., and Smith, K. S.: Novel pyrophosphate-forming acetate kinase from the protist Entamoeba histolytica, Eukaryot. Cell, 11, 1249-1256 (2012).

17. Thaker, T. M., Tanabe, M., Fowler, M. L., Preininger, A. M., Ingram-Smith, C., Smith, K. S., and Iverson, T. M.: Crystal structures of acetate kinases from the eukaryotic pathogens Entamoeba histolytica and Cryptococcus neoformans, J. Struct. Biol., 181, 185-189 (2013).

18. Kitagawa, M., Ara, T., Arifuzzaman, M., Ioka-Nakamichi, T., Inamoto, E., Toyonaga, H., and Mori, H.: Complete set of ORF clones of Escherichia coli ASKA library (a complete set of E. coli K-12 ORF archive): unique resources for biological research, DNA Res., 12, 291-299 (2005).

19. Lipmann, F., and Tuttle, L. C.: A specific micromethod for the determination of acyl phosphates, J. Biol. Chem., 159, 21-28 (1945).

20. Ghetta, A., Matus-Ortega, M., Garcia-Mena, J., Deho, G., Tortora, P., and Regonesi, M. E.: Polynucleotide phosphorylase-based photometric assay for inorganic phosphate, Anal. Biochem., 327, 209-214 (2004).

21. Eriksson, J., Karamohamed, S., and Nyren, P.: Method for real-time detection of inorganic pyrophosphatase activity, Anal. Biochem., 293, 67-70 (2001).

22. Bradford, M. M.: Rapid and sensitive method for quantitation of 
microgram quantities of protein utilizing principle of protein-dye binding, Anal. Biochem., 72, 248-254 (1976).

23. Niesen, F. H., Berglund, H., and Vedadi, M.: The use of differential scanning fluorimetry to detect ligand interactions that promote protein stability, Nat. Protoc., 2, 2212-2221 (2007).

24. Laemmli, U. K.: Cleavage of structural proteins during the assembly of the head of bacteriophage T4, Nature, 227, 680-685 (1970).

25. Altschul, S. F., Madden, T. L., Schaffer, A. A., Zhang, J., Zhang, Z., Miller, W., and Lipman, D. J.: Gapped BLAST and PSI-BLAST: a new generation of protein database search programs, Nucleic. Acids. Res., 25, 3389-3402 (1997).

26. Kaminuma, E., Mashima, J., Kodama, Y., Gojobori, T., Ogasawara, O., Okubo, K., Takagi, T., and Nakamura, Y.: DDBJ launches a new archive database with analytical tools for next-generation sequence data, Nucleic. Acids. Res., 38, D33-38 (2010). 


\section{FIGURES}

432

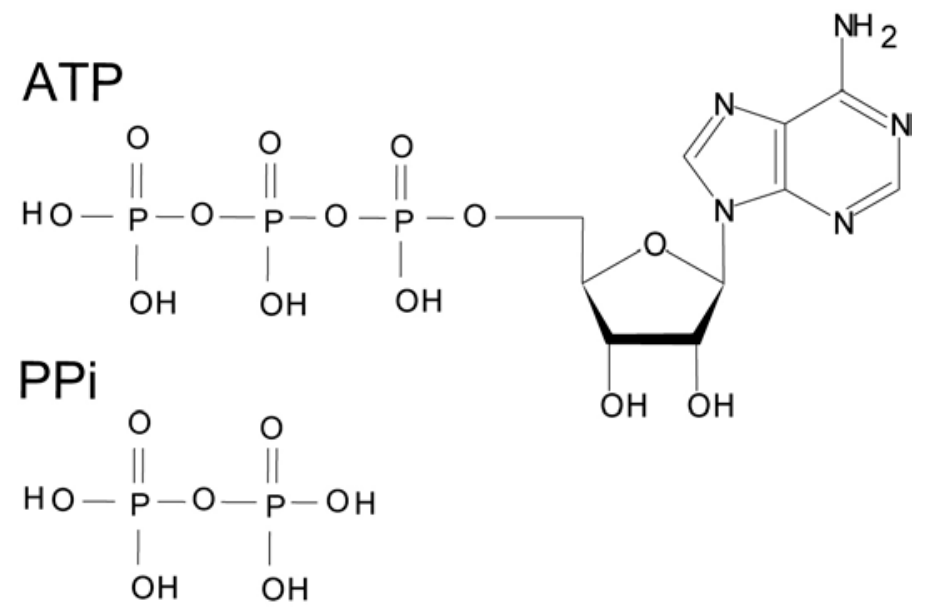

433

434 Fig. 1. Structures of ATP and PPi.

435 
mthAK

ATP-ecoAK

$\mathrm{PPi}$-ehiAK

mthAK

ATP-ecoAK

$\mathrm{PPi}$-ehiAK

mthAK

ATP-ecoAK

$\mathrm{PPi}$-ehiAK

mthAK

ATP-ecoAK

$\mathrm{PPi}$-ehiAK

mthAK

ATP-ecoAK

PPi-ehiAK

mthAK

ATP-ecoAK

$\mathrm{PPi}$-ehiAK

mthAK

ATP-ecoAK

PPi-ehiAK

Fig. 2. Multiple alignment of the primary sequences of mthAK, ATP-ecoAK, and

PPi-ehiAK. Multiple alignment of the mthAK (Uniprot, P38502), ATP-ecoAK

conducted using ClustalW2 (26). The 12 residues located within 4.0 Å of ADP in the tertiary structure of mthAK complexed with acetate and $\mathrm{ADP}-\mathrm{AlF}_{3}$ (9) are shown in bold. Among these 12 residues, the 5 candidate residues for determination of phosphoryl donor specificity are denoted by arrowheads. Key

444 residues involved in catalysis in mthAK (Asn-7, Arg-91, Asp-148, His-180, 445 Arg-241, and Glu-384) (8-10) are conserved in ATP-ecoAK and PPi-ehiAK and 
446 are boxed. Identical residues are denoted by an asterisk $\left(^{*}\right)$, strongly conserved 447 residues by a colon (:), and weakly conserved residues by a period (.). Numbers 448 above the sequences refer to mthAK and numbering for each protein is shown on 449 the right.

A

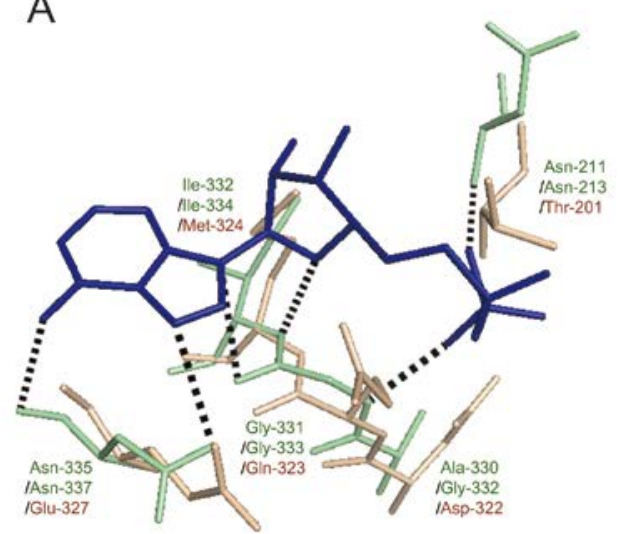

B

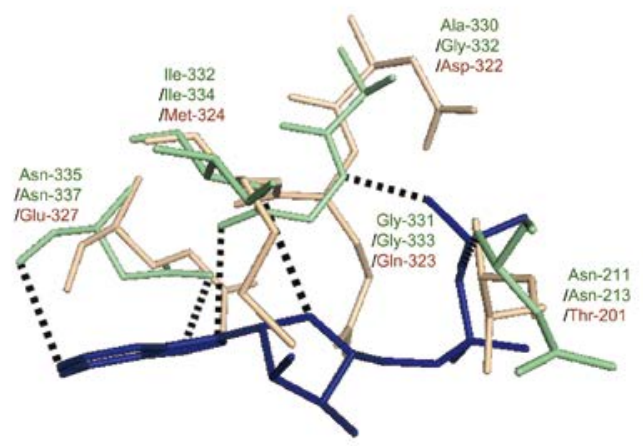

453

454 Fig. 3. Superposition of the substrate-binding site structures of mthAK and 455 PPi-ehiAK. A, Superposition of the substrate-binding sites of PPi-ehiAK (PDB, 456 4H0O) (17) and mthAK complexed with acetate and $\mathrm{ADP}_{-} \mathrm{AlF}_{3}$ (PDB, 1TUY) (9). 457 Amino acid residues of mthAK are shown in green, ADP in mthAK is in deep 458 blue, and PPi-ehiAK is in light orange. Residue names and numbers are shown as 459 mthAK (green)/ATP-ecoAK (green)/PPi-ehiAK (pink). Hydrogen bonds in 460 mthAK are shown as dashed lines. B, Overhead view of A. Superposition of AKs 461 was performed using PyMOL. 
Table 1. Kinetic constants of AKs. ${ }^{\mathrm{a}}$

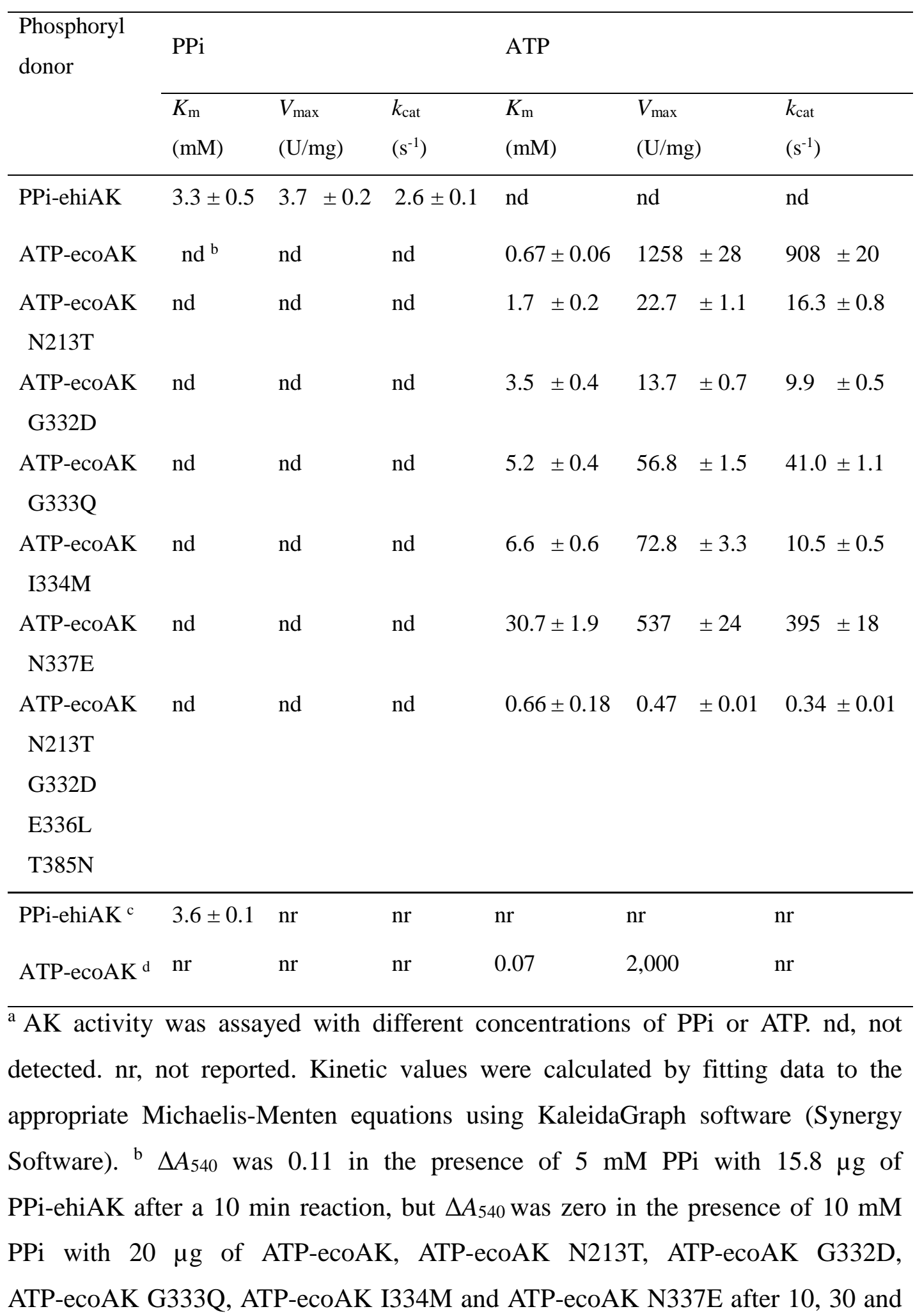


60 min; thus AK activities of ATP-ecoAK and variant ATP-ecoAK toward PPi were regarded as not detected (nd). $\Delta A_{540}$ was 0.036 in the presence of $10 \mathrm{mM}$ ATP with $6.4 \times 10^{-3} \mu \mathrm{g}$ of ATP-ecoAK after a $10 \mathrm{~min}$ reaction, but $\Delta A_{540}$ was zero in the presence of $5 \mathrm{mM}$ ATP with $15.8 \mu \mathrm{g}$ of PPi-ehiAK after 10, 30 and $60 \mathrm{~min}$; thus AK activities of PPi-ehiAK toward ATP were regarded as not detected. ${ }^{\mathrm{c}}$ Data from a previous report (16). ${ }^{\mathrm{d}}$ Data from a previous report (7). 
Table 2. Differences in $T_{\mathrm{m}}$ of AKs in the absence and presence of ATP and PPi.

\begin{tabular}{lcccc}
\hline Enzyme & \multicolumn{2}{l}{$\Delta T_{\mathrm{m} \text { AтP }}\left({ }^{\circ} \mathrm{C}\right)$} & \multicolumn{2}{l}{$\Delta T_{\mathrm{m} \text { PPi }}\left({ }^{\circ} \mathrm{C}\right)$} \\
\hline PPi-ehiAK & nd & & 1.7 & \pm 0.2 \\
ATP-ecoAK & $12.5 \pm 0.9$ & 4.4 & \pm 0.6 \\
ATP-ecoAK N213T & $1.9 \pm 0.1$ & 1.7 & \pm 0.6 \\
ATP-ecoAK G332D & $0.22 \pm 0.18$ & 2.0 & \pm 0.1 \\
ATP-ecoAK G333Q & $2.7 \pm 0.1$ & 3.7 & \pm 0.2 \\
ATP-ecoAK I334M & $1.3 \pm 0.2$ & 3.3 & \pm 0.2 \\
ATP-ecoAK N337E & $1.0 \pm 0.1$ & 3.4 & \pm 0.3 \\
ATP-ecoAK N N213T & $0.73 \pm 0.20$ & 2.7 & \pm 0.2 \\
G332D E338L T385N & & & &
\end{tabular}

Differences in $T_{\mathrm{m}}$ of AKs in the absence and presence of ATP and PPi ( $\Delta T_{\mathrm{m} \text { ATP }}$ and $\left.\Delta T_{\mathrm{m} \mathrm{PPi}}\right)$ are shown. ATP was used at $1.0 \mathrm{mM}$ and PPi at $5.0 \mathrm{mM}$. Means and standard deviations of three independent experiments are shown. nd, not detected. 
Table 3. Distribution of the 5 candidate residues in AK sequences in a database search. $^{\text {a }}$

\begin{tabular}{lll}
\hline $\begin{array}{l}\text { Candidate residues of } \\
\text { mthAK/ATP-ecoAK/PPi-eh }\end{array}$ & $\begin{array}{l}\text { mthAK/ATP-ecoAK-spe } \\
\text { cific residues }\end{array}$ & $\begin{array}{l}\text { PPi-ehiAK-specific } \\
\text { residues }\end{array}$ \\
\hline Asn-211/Asn-213/Thr-201 & $\begin{array}{l}\text { Asn } \\
(2,100 / 80 \%)^{\text {b }}\end{array}$ & $\begin{array}{l}\text { Thr } \\
(2 / 0.08 \%)\end{array}$ \\
Ala-330/Gly-332/Asp-322 & $\begin{array}{l}\text { Ala or Gly } \\
(2,584 / 98 \%)\end{array}$ & Asp \\
& Gly & Gln \\
Gly-331/Gly-333/Gln-323 & $(2,581 / 98 \%)$ & $(2 / 0.08 \%)$ \\
& Ile & Met \\
Ile-332/Ile-334/Met-324 & $(1,752 / 67 \%)$ & $(78 / 3 \%)$ \\
Asn-335/Asn-337/Glu-327 & Asn & Glu \\
& $(2,101 / 80 \%)$ & $(10 / 0.38 \%)$
\end{tabular}

${ }^{a}$ Distribution of the 5 candidate residues of mthAK/ATP-ecoAK/PPi-ehiAK (Asn-211/Asn-213/Thr-201, Ala-330/Gly-332/Asp-322, Gly-331/Gly-333/Gln-323, Ile-332/Ile-334/Met-324, and Asn-335/Asn-337/Glu-327) in a database search of AKs. A total of 2,625 PPi-ehiAK homologs were found using BLASTP (25) on the GenomeNet server (http://www.genome.jp). ${ }^{\text {b }}$ Number and percentage (\%) of PPi-ehiAK homologs possessing the indicated amino acid at the position of the candidate residue. 


\section{Supplementary information}

Table S1. Interactions between mthAK and ADP ${ }^{\mathrm{a}}$

\begin{tabular}{|c|c|c|c|c|c|c|c|}
\hline \multicolumn{4}{|c|}{ Hydrogen bonds } & \multicolumn{4}{|c|}{ van der Waals contacts } \\
\hline ADP atoms & \multicolumn{2}{|c|}{ Target atoms } & Distance $(\AA)$ & ADP atoms & \multicolumn{2}{|c|}{ Target atoms } & Distance $(\AA)$ \\
\hline \multicolumn{8}{|l|}{$\beta$-phosphate } \\
\hline $\mathrm{O} 2 \mathrm{~B}$ & Asn-211 & $\mathrm{N}$ & 2.7 & & & & \\
\hline О3В & Gly-331 & $\mathrm{N}$ & 2.6 & & & & \\
\hline \multicolumn{8}{|l|}{ Ribose } \\
\hline $\mathrm{O} 2^{\prime}$ & Phe- $284^{c}$ & $\mathrm{~N}$ & 3.3 & C1' & Gly-331 ${ }^{b}$ & C & 3.7 \\
\hline O3' & Asp- $283^{c}$ & OD2 & 3.3 & & Ile-332 & CG1 & 3.5 \\
\hline O4' & Ile-332 ${ }^{\mathrm{b}}$ & $\mathrm{N}$ & 3.6 & $\mathrm{C}^{\prime}$ & Phe- $284^{\mathrm{C}}$ & СB & 3.9 \\
\hline \multicolumn{8}{|l|}{ Adenine } \\
\hline N3 & $\operatorname{Arg}-285^{C}$ & $\mathrm{~N}$ & 3.5 & $\mathrm{C} 2$ & $\operatorname{Arg}-285^{\mathrm{C}}$ & CA & 3.6 \\
\hline \multirow[t]{2}{*}{ N6 } & Asn-335 & $\mathrm{O}$ & 3.4 & & & CB & 3.9 \\
\hline & Arg-362 ${ }^{\mathrm{d}}$ & $\mathrm{NH}_{2}$ & 3.2 & & Ser- $336^{\mathrm{d}}$ & СВ & 3.8 \\
\hline N7 & Asn-335 & ND2 & 3.2 & C5 & Asn- $335^{b}$ & CB & 3.8 \\
\hline N9 & Gly-331 & $\mathrm{O}$ & 3.4 & & & & \\
\hline
\end{tabular}

${ }^{a}$ Interactions between ADP and residues of mthAK B chain. ${ }^{\mathrm{b}}$ Five candidate residues specific to PPi-ehiAK. ${ }^{\mathrm{c}}$ Five residues conserved in mthAK, ATP-ecoAK, and PPi-ehiAK.

${ }^{\mathrm{d}}$ Residues not conserved. 
Table S2. AK activity in the PPi-forming direction using $\mathrm{Pi}$ as a phosphoryl acceptor ${ }^{\text {a }}$.

\begin{tabular}{llc}
\hline Enzyme & PPi $(\boldsymbol{\mu M})$ \\
\hline PPi-ehiAK & 3.1 \\
ATP-ecoAK & nd ${ }^{\mathrm{b}}$ \\
ATP-ecoAK & N213T & nd \\
ATP-ecoAK & G332D & nd \\
ATP-ecoAK & G333Q & nd \\
ATP-ecoAK & I334M & nd \\
ATP-ecoAK & N337E & nd \\
\hline
\end{tabular}

${ }^{\mathrm{a}}$ AK activity in the PPi-forming direction was assayed as described in the Materials and Methods. After reaction for 20 min with $1.4 \times 10^{-2} \mu \mathrm{g}$ of PPi-ehiAK, the reaction mixture was diluted 50-fold with distilled water and RLU was measured. PPi at $1.0 \mu \mathrm{M}$ gave $\Delta$ RLU of 955,937. nd, not detected.

${ }^{\mathrm{b}} \Delta \mathrm{RLU}$ was zero with 9.2, 3.1, 2.4, 5.8, 2.9, and 3.1 $\mu$ g of ATP-ecoAK, ATP-есоAK N213T, ATP-есоAK G332D, ATP-есоAK G333Q, ATP-ecoAK I334M and ATP-ecoAK N337E, respectively, after the reaction for $20 \mathrm{~min}$; thus, AK activities in the PPi-forming direction of ATP-ecoAK and these mutated ATP-ecoAKs using PPi were regarded as not detected (nd). 
Table S3. Residues interacting with Thr-201 and Asp-322 in PPi-ehiAK .

\begin{tabular}{|c|c|c|c|c|c|c|}
\hline \multicolumn{4}{|c|}{ Hydrogen bonds } & \multicolumn{3}{|c|}{ van der Waals contacts } \\
\hline & \multicolumn{2}{|c|}{ Target atoms } & \multicolumn{2}{|l|}{ Distance $(\AA)$} & Target atoms & \multirow[t]{2}{*}{ Distance $(\AA)$} \\
\hline Thr-201 & & & & & & \\
\hline $\mathrm{O}$ & Gly-202 & $\mathrm{N}$ & 3.4 & & & \\
\hline \multicolumn{7}{|c|}{ Asp-322 } \\
\hline $\mathrm{O}$ & Leu-326 ${ }^{c}$ & $\mathrm{~N}$ & 3.4 & & & \\
\hline \multirow[t]{2}{*}{ OD1 } & His-198 & ND1 & 3.3 & CA & Asn-376 $\quad$ CG & 3.9 \\
\hline & & NE2 & 2.3 & СВ & Leu-199b $\quad$ C & 3.4 \\
\hline \multirow[t]{2}{*}{ OD2 } & Leu-199 & $\mathrm{O}$ & 3.6 & & Gly-200 $\quad$ CA & 3.9 \\
\hline & Gly-200 & $\mathrm{N}$ & 3.3 & CG & Leu-199 ${ }^{b} \quad$ C & 3.9 \\
\hline $\mathrm{N}$ & Asn-376 & OD1 & 3.1 & & Gly-200 $\quad$ CA & 3.4 \\
\hline \multicolumn{7}{|c|}{$\begin{array}{l}\text { a The residues interacting with Thr-201 and Asp-322 in PPi-ehiAK. Thr-201 and } \\
\text { Asp-322 are expected to be around the phosphate group. b Four residues } \\
\text { conserved in the primary structures of mthAK, ATP-ecoAK, and PPi-ehiAK. c }\end{array}$} \\
\hline
\end{tabular}


Table S4. Distribution of the 5 candidate residues.

\begin{tabular}{|c|c|c|c|c|c|c|}
\hline \multirow[b]{2}{*}{ Homolog name } & \multirow[b]{2}{*}{ Species name } & \multicolumn{5}{|c|}{ Amino-acid residue corresponding to } \\
\hline & & Glu-327 & Thr-201 & $\begin{array}{l}\text { Asp-322 } \\
\text { f PPi-ehiA }\end{array}$ & Gln-323 & Met-324 \\
\hline EDI_237080 a & $\begin{array}{l}\text { Entamoeba } \\
\text { dispar }\end{array}$ & Glu & Thr & Asp & Gln & Met \\
\hline GM21_0328 a & $\begin{array}{l}\text { Geobacter } \\
\text { sp. M21 }\end{array}$ & Glu & Asn & Tyr & Gly & Glu \\
\hline Gbem_0348 ${ }^{\text {a }}$ & $\begin{array}{l}\text { Geobacter } \\
\text { bemidjiensis }\end{array}$ & Glu & Asn & Tyr & Gly & Glu \\
\hline GM18_0425 a & $\begin{array}{l}\text { Geobacter } \\
\text { sp. M18 }\end{array}$ & Glu & Asn & Tyr & Gly & Glu \\
\hline Tpet_1041 ${ }^{\mathrm{a}}$ & $\begin{array}{l}\text { Thermotoga } \\
\text { petrophila }\end{array}$ & Glu & Ser & Gly & Gly & Met \\
\hline Tnap_1061 ${ }^{\text {a }}$ & $\begin{array}{l}\text { Thermotoga } \\
\text { naphthophila }\end{array}$ & Glu & Ser & Gly & Gly & Met \\
\hline TRQ2_1071 ${ }^{\mathrm{a}}$ & $\begin{array}{l}\text { Thermotoga sp. } \\
\text { RQ2 }\end{array}$ & Glu & Ser & Gly & Gly & Met \\
\hline Tmari_1762 ${ }^{\mathrm{a}}$ & $\begin{array}{l}\text { Thermotoga } \\
\text { maritima }\end{array}$ & Glu & Ser & Gly & Gly & Met \\
\hline TM1754 ${ }^{\mathrm{a}}$ & $\begin{array}{l}\text { Thermotoga } \\
\text { maritima }\end{array}$ & Glu & Ser & Gly & Gly & Met \\
\hline АТР-ecoAK & E. coli & Asn & Asn & Gly & Gly & Ile \\
\hline mthAK & M. thermophila & Asn & Asn & Ala & Gly & Ile \\
\hline
\end{tabular}

${ }^{a}$ Proteins in KEGG database (http://www.genome.jp/kegg/).

b Y75_p2262 in KEGG database. 
CGCGCGGCAGCCATATGTCTAATGTGCTGATTTTCAACGTAGGATCTAGTTCCCTCACATATAAA GTGTTTTGTAGCGACAACATCGTCTGTAGTGGCAAATCCAATCGCGTGAATGTGACTGGTACTG AGAAACCGTTTATCGAACATCACTTAAACGGCCAAATCATTAAGATAGAAACCCCAATCCTCAA CCATCCACAGGCTGCAAAACTGATTATCCAGTTCCTGAAAGAGAACCACATCTCTATTGCCTTT GTAGGCCATCGCTTTGTTCATGGCGGGTCTTACTTTAAGAAATCAGCCGTCATTGACGAAGTCG TGCTGAAAGAACTGAAAGAATGCCTGCCTTTAGCGCCCATTCATAACCCGAGCTCTTTCGGAG TTATCGAAATCTCGATGAAAGAACTGCCAACCACTCGTCAGTATGTGGCGATCGATACGGCGTT CCATTCGACCATTTCCCAAGCTGAACGCACCTATGCGATTCCGCAGCCGTATCAATCGCAGTAT CTGAAATTCGGCTTTCACGGCCTGTCATACGAATACGTGATCAACTCGTTGAAGAATGTCATTG ACGTAAGTCACAGCAAAATCATTGCATGCCATCTTGGTACAGGTGGCTCCTCATGCTGCGGCAT TGTGAATGGGAAATCGTTTGATACGAGTATGGGTAACAGCACACTTGCTGGTCTGGTGATGTCA ACGCGTTGTGGGGATATTGATCCCACCATTCCGATTGATATGATCCAGCAAGTGGGTATTGAGA AAGTTGTCGATATTCTCAACAAGAAAAGCGGCTTGCTTGGGGTCAGTGAACTGAGCAGCGATA TGCGCGATATTTTGCACGAAATCGAAACCCGTGGTCCTAAAGCCAAAACGTGCCAACTGGCCT TTGATGTTTACATCAAACAGCTGGCGAAAACCATCGGAGGCCTGATGGTTGAGATCGGCGGTC TTGACTTACTGGTGTTCACCGATCAGATGGGTCTGGAAGTATGGCAAGTTCGGAAAGCGATATG CGACAAAATGAAATTTCTGGGCATTGAGTTGGACGATAGCCTCAATGAGAAGTCCATGGGTAA GAAAATTGAATTCCTGACGATGCCGAGCAGCAAGGTACAGGTTTGTGTTGCACCGAATGACGA GGAATTAGTCATACTGCAGAAAGGGAAAGAATTATTCCAGTTTTAACTCGAGCACCACCACC

Fig. S1. The sequence of PPi-ehiAK with optimized codon usage for E. coli. The optimized PPi-ehiAK gene (KEGG ID; ehi: EHI_170010) was synthesized at Operon. The NdeI site (CATATG) and XhoI site (CTCGAG) are underlined. The start codon (ATG) and stop codon (TAA) are shown in bold. 


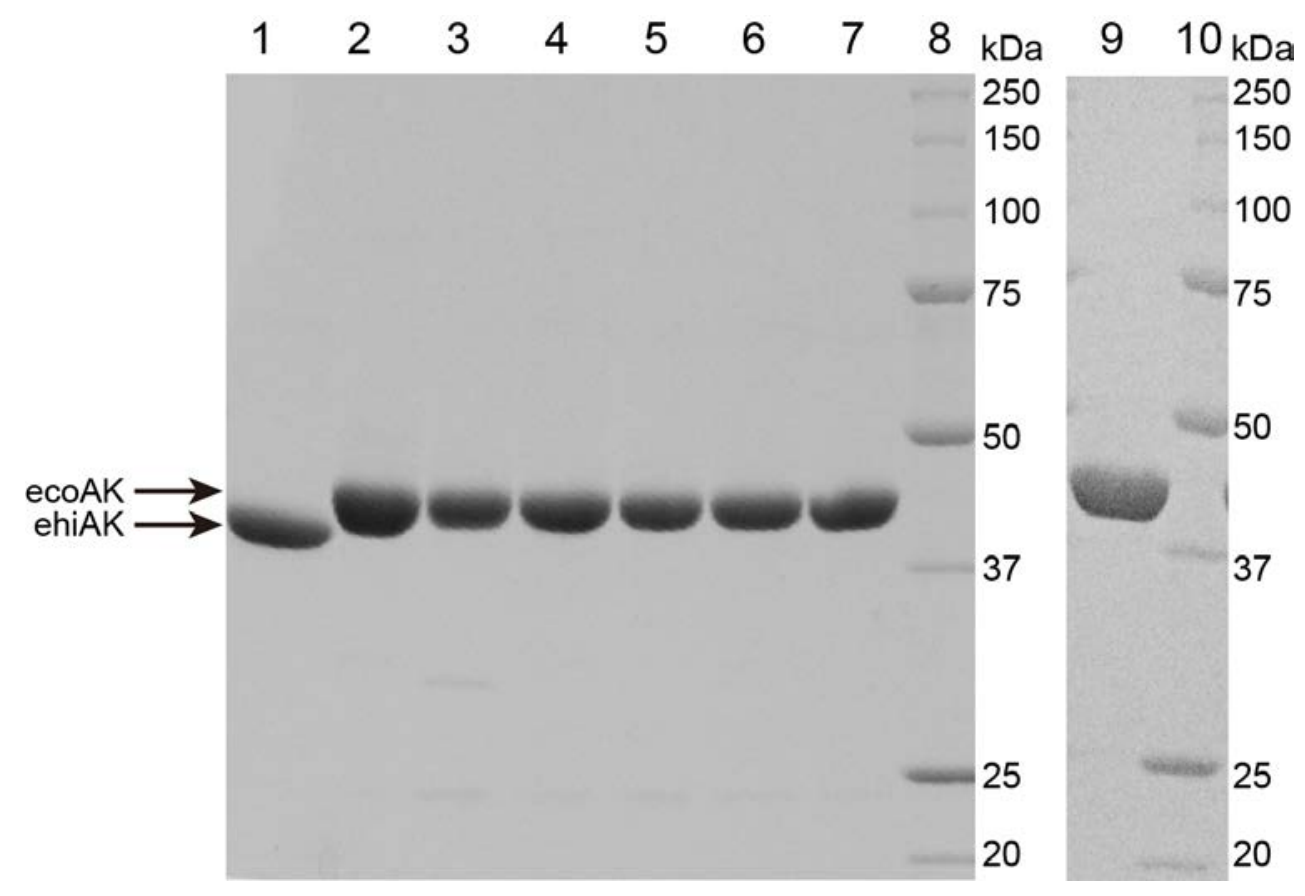

Fig. S2. SDS-PAGE of purified AKs. Lanes 1-7, 9: purified PPi-ehiAK, ATP-ecoAK, ATP-ecoAK N213T, ATP-ecoAK G332D, ATP-ecoAK G333Q, ATP-есоAK I334M, ATP-ecoAK N337E, and ATP-ecoAK N213T G332D E336L T385N (5.0 $\mu \mathrm{g})$. Arrows indicate positions of purified AKs. Lanes 8 and 10: protein markers (Bio-Rad Laboratories, Hercules, CA). 

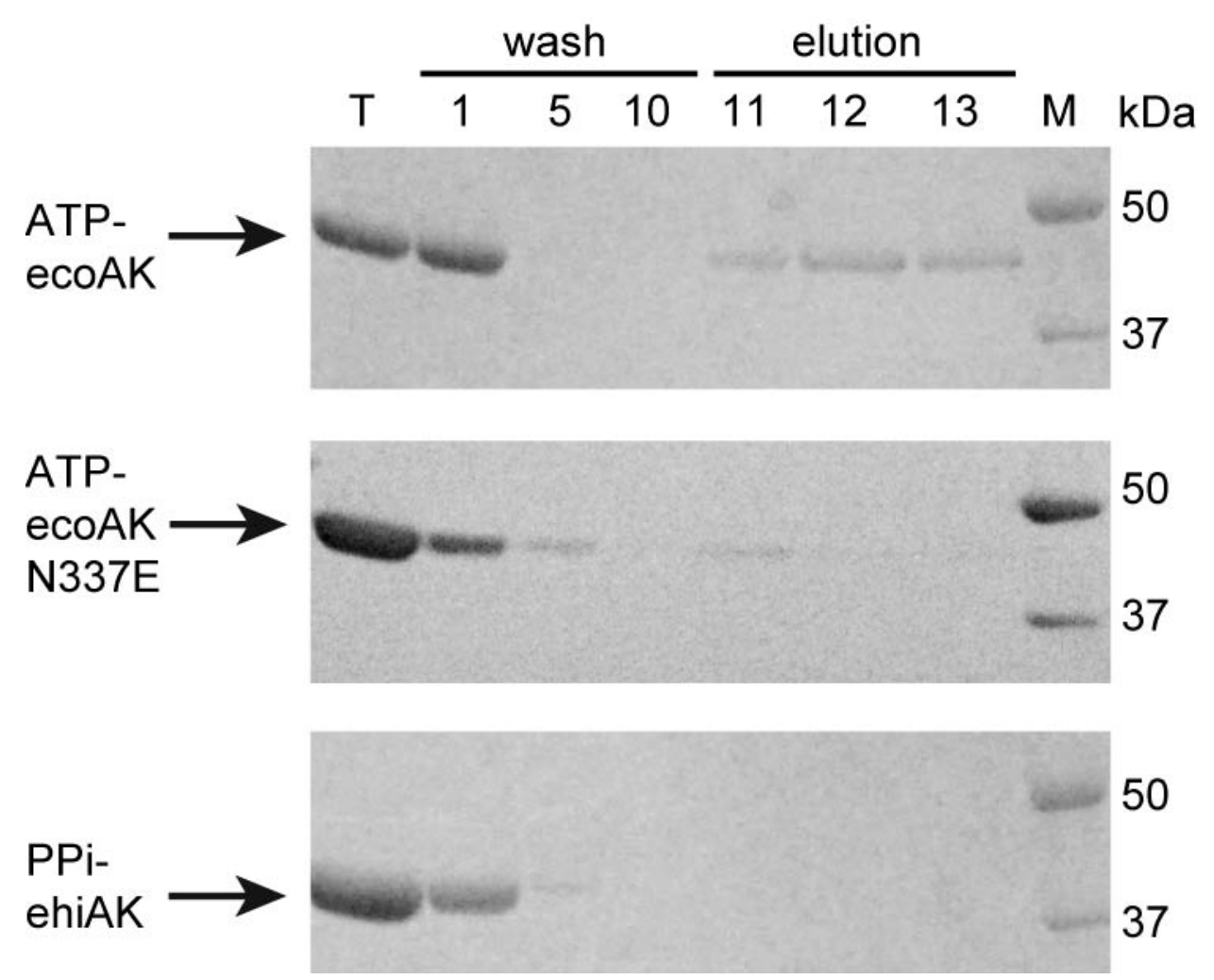

Fig. S3. SDS-PAGE of AKs eluted from an ATP-agarose column. Lane T: flow-through fraction; Lanes 1, 5, and 10: Fractions washed with $10 \mathrm{mM}$ Tris-HCl (pH 8.0) containing 10 $\mathrm{mM} \mathrm{MgCl} 2$ or $20 \mathrm{mM}$ HEPES-NaOH (pH 7.5) containing $10 \mathrm{mM} \mathrm{MgCl}_{2}$; Lanes 11, 12, and 13: Fractions eluted with $50 \mathrm{mM}$ ATP containing $10 \mathrm{mM} \mathrm{MgCl}_{2}$; Lane $\mathrm{M}$ : protein markers. Lane numbers correspond to the numbers of fractions (200 $\mu \mathrm{L}$ each). Arrows indicate the positions of AKs. 

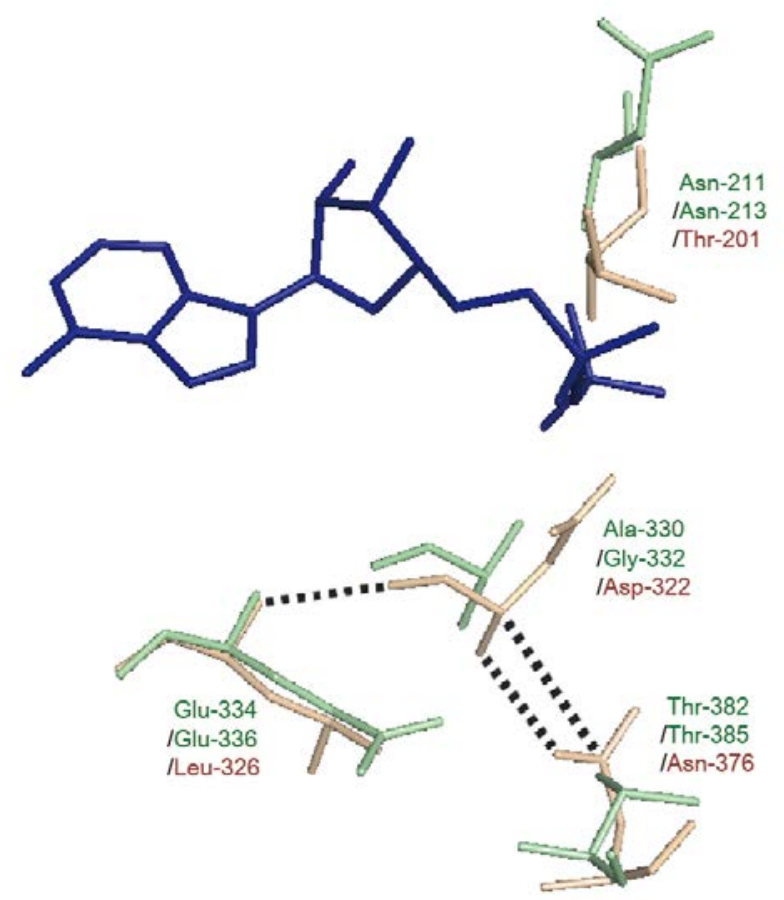

Fig. S4. Superposition of mthAK and PPi-ehiAK around the phosphate group of ADP. Superposition of the tertiary structure of PPi-ehiAK (PDB, 4H0O) (1) and that of mthAK complexed with acetate and $\mathrm{ADP}-\mathrm{AlF}_{3}(\mathrm{PDB}, 1 \mathrm{TUY})$ (2). Amino acid residues of mthAK are shown in green; ADP in mthAK is in deep blue, and PPi-ehiAK is in light orange. The residue names and numbers are shown for mthAK (green)/ATP-ecoAK (green)/PPi-ehiAK (pink). Hydrogen bonds in PPi-ehiAK are shown as dashed lines. Superposition of AKs was performed using PyMOL. 

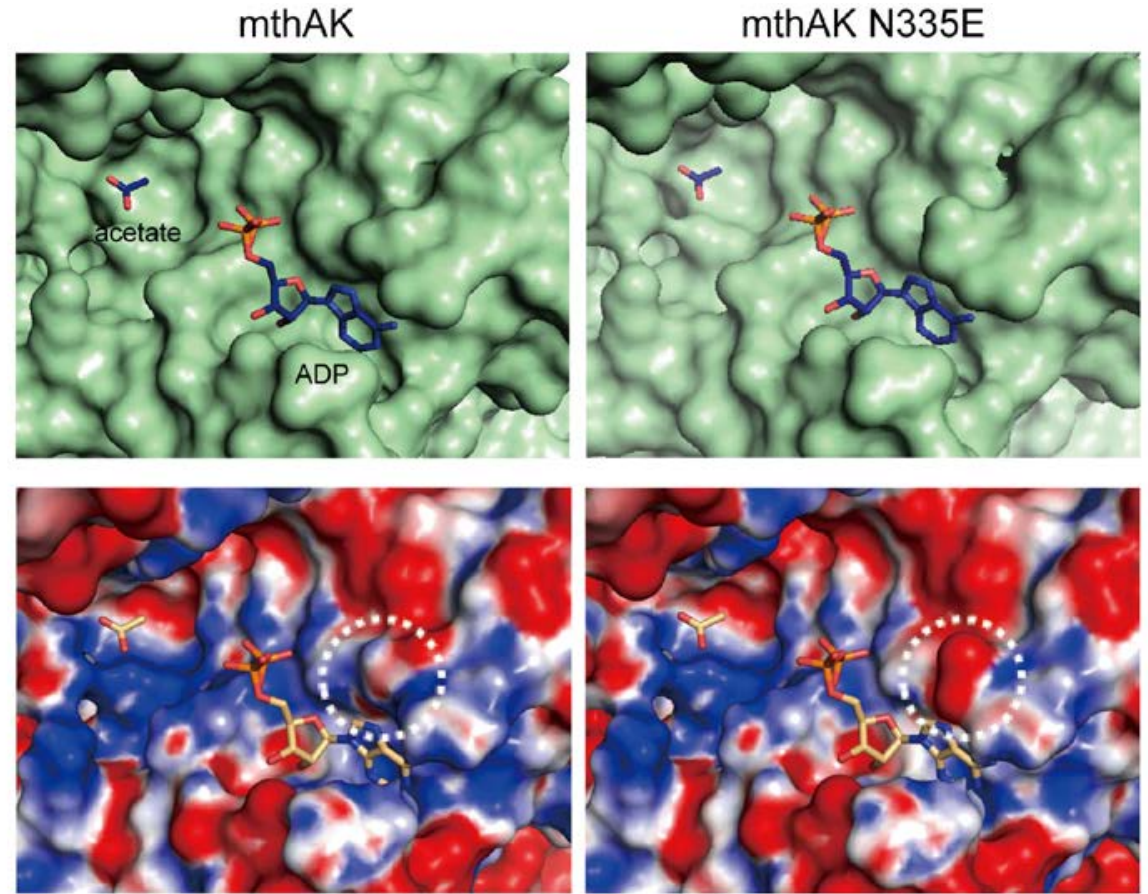

$$
K_{\mathrm{m}}(\mathrm{mM}) \quad 0.67 \pm 0.06
$$$$
30.7 \pm 1.9
$$

$V_{\max }(\mathrm{U} / \mathrm{mg})$

$1,258 \pm 28$

$537 \pm 24$

Fig. S5. ADP-binding sites of mthAK and mthAK N335E. Molecular surfaces of ADP-binding sites of mthAK (left) and mthAK N335E (right) are shown in the upper panels. Electrostatic surfaces of the AKs are shown in the lower panels. Positive and negative charges at pH 7.0 are colored in blue and red, respectively, and were calculated using APBS (3). The position around Asn-335 is denoted by a white dotted circle. $K_{\mathrm{m}}$ and $V_{\max }$ for ATP of ATP-ecoAK and ATP-ecoAK N337E are shown under the respective figures. 


\section{References}

1. Thaker, T. M., Tanabe, M., Fowler, M. L., Preininger, A. M., Ingram-Smith, C., Smith, K. S., and Iverson, T. M.: Crystal structures of acetate kinases from the eukaryotic pathogens Entamoeba histolytica and Cryptococcus neoformans, J. Struct. Biol., 181, 185-189 (2013).

2. Gorrell, A., Lawrence, S. H., and Ferry, J. G.: Structural and kinetic analyses of arginine residues in the active site of the acetate kinase from Methanosarcina thermophila, J. Biol. Chem., 280, 10731-10742 (2005).

3. Baker, N. A., Sept, D., Joseph, S., Holst, M. J., and McCammon, J. A.: Electrostatics of nanosystems: application to microtubules and the ribosome, Proc. Natl. Acad. Sci. USA., 98, 10037-10041 (2001). 